\title{
Channeling effect in electronic spectra produced by grazing impact of fast protons on insulator surfaces
}

\author{
C. D. Archubi and M. S. Gravielle \\ Instituto de Astronomía y Física del Espacio, Casilla de Correo 67, Sucursal 28, C1428ZAA Buenos Aires, Argentina \\ and Departamento de Física, Facultad de Ciencias Exactas y Naturales, Universidad de Buenos Aires, Buenos Aires, Argentina
}

(Received 17 July 2009; published 10 September 2009)

\begin{abstract}
Electron emission due to grazing scattering of fast protons from $\mathrm{LiF}$ and $\mathrm{KCl}$ surfaces is studied under axial channeling conditions. The differential electron emission probability is calculated within a distorted-wave formalism, taking into account realistic channeled trajectories. For electronic spectra along low-index crystallographic directions, we find that channeling effects on the electron emission yield depend strongly on the penetration rate of projectiles into the solid.
\end{abstract}

DOI: 10.1103/PhysRevA.80.034901

PACS number(s): 34.35.+a, 34.50.Fa

\section{INTRODUCTION}

For grazing impact of projectiles on monocrystalline surfaces, it is possible to distinguish two regimes, usually known as planar and axial surface channeling [1]. Both regimes, associated with incidence along random and lowindex directions of the crystal, respectively, present different characteristics. While in the case of planar channeling, the incident particle is specularly reflected from an effectively flat surface, under axial surface channeling conditions the projectile is steered by rows of surface ions [2], which affects not only the projectile path but also the electronic excitation processes produced during the collision.

In particular, for atoms or ions colliding along low-index crystallographic directions, an enhancement of the electron emission yield is experimentally observed $[3,4]$. Two different explanations for this increment of the electron emission probability under axial incidence have been proposed. While a recent experimental work attributes an important role to the fact that electron spectra may be recorded in coincidence or not with specularly reflected projectiles [5], other researches show that even for those projectiles recorded in coincidence there is an effect due to the axial channeling [6-8]. To shed light on this question, in this Brief Report we study the influence of the projectile trajectory on electron distributions originated by swift protons reflected from the topmost atomic layer of insulator surfaces.

\section{THEORETICAL METHOD}

When a fast proton impinges grazingly on an ionic surface, it moves along the trajectory mainly as a bare ion [9]. As a consequence of the localized character of the electronic density we can assume that electron emission is caused by a sequence of binary collisions of the projectile with surface ions placed at the first atomic layer, each of them contributing on its own to the electronic spectrum. Then, the differential probability of electron emission with momentum $\vec{k}$ is obtained as

$$
\frac{d P_{i}}{d \vec{k}}=\delta \int_{-\infty}^{\infty} d x \int_{-\infty}^{\infty} d y P_{i \vec{k}}^{(a t)}(\rho(x, y)),
$$

where $P_{i k}^{(a t)}$ is the differential probability of atomic ionization from the initial state $i$, as a function of the impact parameter $\rho$, and $\delta$ is the surface atomic density, which is considered a constant. The impact parameter $\rho$ depends on the position $(x, y)$ of each surface ion as $\rho(x, y)=\sqrt{y^{2}+z(x)^{2}}$, where $x$ and $y$ correspond to the coordinates along and perpendicular to the incidence direction, respectively, on the surface plane, and $z(x)$ is the coordinate of the projectile trajectory normal to the surface [10].

The probability of atomic ionization $P_{i \vec{k}}^{(a t)}$ is evaluated within the continuum-distorted-wave-eikonal-initial-state (CDW-EIS) approximation $[11,12]$, which includes the final electron interactions with both the passing projectile and the effective charge left in the surface after ionization. The CDW-EIS $T$-matrix element reads $T_{i f}=\left|\left\langle\chi_{f}^{C D W^{-}}\left|W_{f}^{\dagger}\right| \chi_{i}^{E^{+}}\right\rangle\right|$, where $\chi_{f}^{C D W^{-}}$and $\chi_{i}^{E^{+}}$are the CDW and eikonal distortedwave functions, with the proper asymptotic conditions in the final and initial channels, respectively, and $\mathrm{W}_{f}^{\dagger}$ is the final perturbative potential. Details of these CDW-EIS calculations can be found in Ref. [12].

The classical path of the projectile is evaluated by using the punctual model of Ref. [10] which allows us to incorporate the crystallographic effects on the trajectory. Within this punctual model, for a given incidence direction the different projectile trajectories can be labeled by means of the vector $\vec{R}_{0}=\left(x_{0}, y_{0}, z_{0}\right)$, which identifies the initial position of the incident particle. The coordinate $z_{0}$ represents the initial distance of the projectile to the surface, while the coordinates $\left(x_{0}, y_{0}\right)$ determine its initial position on the surface plane. In this Brief Report, for a fixed distance $z_{0}$ far from the surface we consider random initial positions $\left(x_{0}, y_{0}\right)$ on the surface plane. Hence, the differential emission probability is obtained by averaging $d P_{i} / d \vec{k}$ over the different projectile paths.

\section{RESULTS}

The method is applied to 100 and $500 \mathrm{keV}$ protons impinging grazingly on $\mathrm{LiF}$ and $\mathrm{KCl}$ surfaces, respectively. Owing to the geometry of the collision, associated with grazing incidence, the ionization is mainly produced from the external shells of surface ions; that is, the $L$ shell of the $\mathrm{F}^{-}$ anions and the $K$ shell of the $\mathrm{Li}^{+}$cations for $\mathrm{LiF}$, and the $M$ 
and $O$ shells of $\mathrm{Cl}^{-}$anions and the $L$ shell of the $\mathrm{K}^{+}$cations for $\mathrm{KCl}$.

Classical projectile trajectories were derived from Newton's equations corresponding to the punctual potential of Ref. [10] by using the Runge Kutta method. In the calculation of the projectile-surface interaction, for every position of the projectile we included contributions from the fourthorder nearest-neighbor target ions, which involves four atomic layers of the solid, i.e., $8 \times 8 \times 4$ target ions. In insulators the effect of the dynamic polarization of the surface is partially compensated by the track potential originated by the ionization process [13]. Consequently, the individual projectile-ion potentials were evaluated taking into account only the static contribution. In all the cases 30000 projectiles paths with random initial conditions were considered. As we are interested in electron emission produced by projectiles that do not penetrate either into the bulk or the subsurface region, only the first 1000 paths strictly reflected above the surface were selected from these trajectories to evaluate the emission probability.

We have considered two different types of electron emission according to the method used to record the results: noncoincidence and coincidence emission. In the first case we record all emitted electrons corresponding to nonpenetrating projectiles, while in the second case we take into account only those electrons emitted in coincidence with specularly reflected projectiles. In order to evaluate coincidence emission, the resolution of the detector was assumed to be 0.3 $\mathrm{mrad}$, the same used in the experiment of Ref. [6]. Note that here the noncoincidence case differs from the experimental one, in which the contribution of projectiles penetrating into the solid is also included.

We start studying grazing collisions of $100 \mathrm{keV}$ protons with a $\mathrm{LiF}(001)$ surface for two different impact angles, $0.3^{\circ}$ and $0.7^{\circ}$, both measured with respect to the surface plane. Three incidence directions on the surface plane were analyzed: two of them along the principal axes [110] and [100], respectively, and the third one, considered as a random incidence direction, far apart from low-index crystallographic orientations, corresponding to the azimuthal angle $\varphi_{i}=30^{\circ}$ relative to the [100] axis. We found that while for random incidence all protons are specularly reflected above the surface, under axial channeling conditions the penetration of ions depends on the channel and the incidence angle.

For the lowest-impact angle $\theta_{i}=0.3^{\circ}$ we observe a very low or null penetration rate: only $12 \%$ for incidence along the [100] channel, while for the [110] channel all projectiles are reflected above the surface. In Fig. 1 we show the corresponding noncoincidence electronic spectra for the electron emission angles $\theta_{e}=5,10$, and $30^{\circ}$. Note that the maximum observed for $\theta_{e}=5^{\circ}$ is a consequence of the capture to the continuum peak present in the forward direction. Although at low-electron velocities all the results converge, channeling effects appear as the energy increases. For the most opened [100] channel the emission probability shows an increment of about $30 \%$ with respect to the value corresponding to the random incidence direction, while for the [110] channel this difference is lower than $10 \%$. These relations between channeled and random probabilities hold for the whole angular range of electron ejection, providing similar rates for the

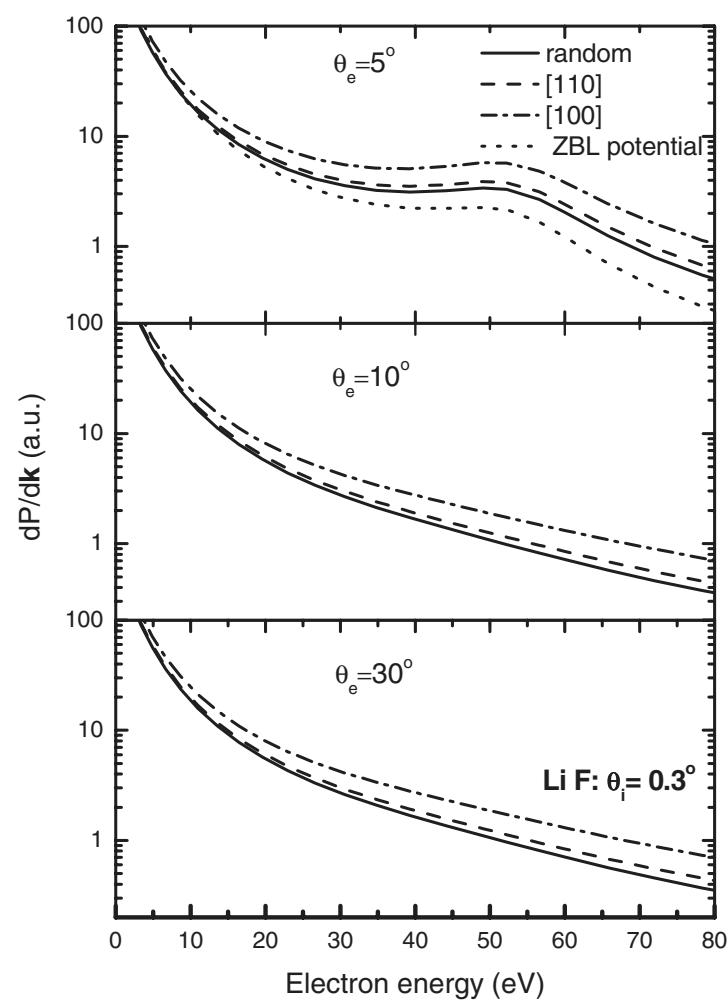

FIG. 1. Emission probability for electrons ejected from a $\mathrm{LiF}(001)$ surface at the emission angles $\theta_{e}=5,10$, and $30^{\circ}$, as a function of the electron energy. The incidence angle is $\theta_{i}=0.3^{\circ}$. Three incidence directions of protons are considered. Full line, random incidence corresponds to a polar angle far from low-index crystallographic orientations; dashed and dashed-dotted lines correspond to the directions of the two principal axes, [110] and [100], as indicated in the figure. Dotted line, results obtained by using a planar ZBL potential to derive the projectile trajectory.

total emission yields. As a reference in Fig. 1, we also plot planar channeling results obtained with a trajectory derived from the Ziegler-Biersack-Littmark (ZBL) potential. We observe that for this glancing incidence angle $\theta_{i}=0.3^{\circ}$ the $\mathrm{ZBL}$ potential leads to electron emission probabilities lower than those derived from the punctual model, even for random incidence.

The observed enhancement of the electron emission yield for the [100] channel is reinforced when we record ejected electrons in coincidence with specularly reflected projectiles. For the impact angle of $0.3^{\circ}$, where the grazing condition is almost fully satisfied, electron emission probabilities for the [100] channel increase when only contributions of specularly reflected projectiles are considered, rising from $30 \%$ to $60 \%$ above the values corresponding to random incidence. Instead, emission probability for the [110] channel stays almost invariable, being similar in coincidence and noncoincidence calculations.

However, the above situation changes completely when the impact angle $\theta_{i}$ augments. Noncoincidence spectra for $\theta_{i}=0.7^{\circ}$ are plotted in Fig. 2 for the same ejection angles as in Fig. 1. In this case the penetration rate is very important: $70 \%$ for the [100] channel and $63 \%$ for the [110] channel. Electronic spectra corresponding to incidence along the main 


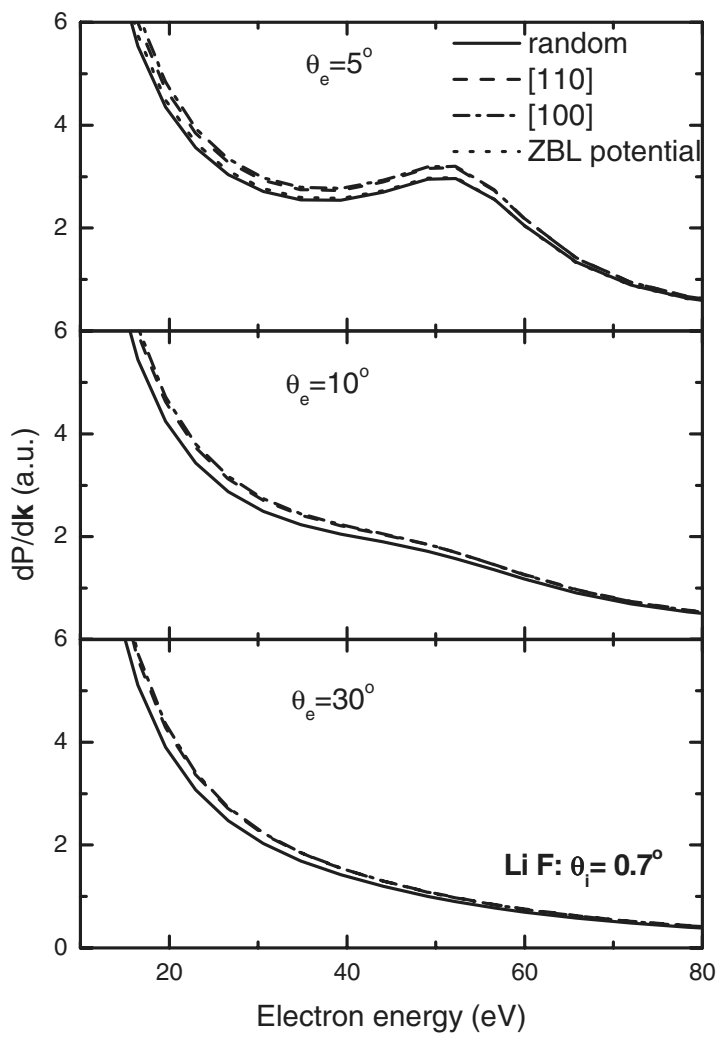

FIG. 2. Similar to Fig. 1 for the incidence angle $\theta_{i}=0.7^{\circ}$.

low-index crystallographic channels display very small differences between them; these axial channeled probabilities run only about $10 \%$ above the random one, which is in this case similar to that provided by the ZBL model. Notice that a linear scale is employed in Fig. 2 in order to distinguish the different curves, all the contributions being slightly lower than the corresponding to Fig. 1. This is due to the fact that the more grazing the collision the longer the distance the projectile remains near to the surface, which increases the electron emission. By recording in coincidence with specularly reflected protons, the electron emission yield for the [100] channel approximates even more to the random values but an increment of the emission probability for the [110] channel is observed.

Similar behaviors are found for grazing collisions of 500 $\mathrm{keV}$ protons with a $\mathrm{KCl}(001)$ surface. In Fig. 3 we show noncoincident electron distributions for the angle $\theta=0.1^{\circ}$, for which the penetration phenomena is negligible and, thus, the grazing condition is completely verified. Note that the maxima displayed in the energy spectra corresponding to the ejection angles $\theta_{e}=10$ and $30^{\circ}$ are associated with the binary collision ridge. Figures show clearly separated curves at intermediate energies. For incidence along the [100] channel differential emission probabilities run more than $20 \%$ above the random values, and this rate increases to $50 \%$ when the detection is made in coincidence with specularly reflected protons. While for the [110] channel emission probabilities calculated with both recording methods are close to the random one, as also observed in the case of Fig. 1. The enhancement of the probability for channel [100] is in qualitative accord with experimental data obtained by Andou et al. [6].

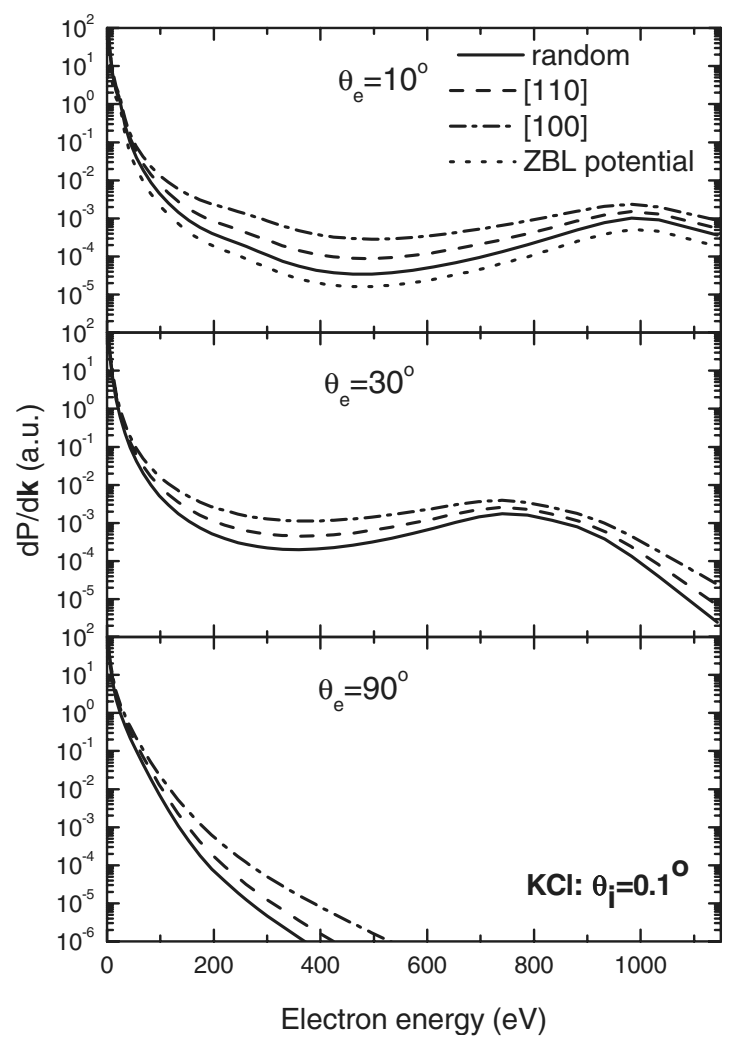

FIG. 3. Similar to Fig. 1 for protons impinging on a $\mathrm{KCl}(001)$ surface with the incidence angle $\theta_{i}=0.1^{\circ}$.

However, for a higher-impact angle, such as $\theta_{i}=0.28^{\circ} \mathrm{em}-$ ployed in the experiment, the penetration rate derived with the punctual model is important, higher than 70\%. Then, axial channeling results obtained with both recording methods show much small differences with random values, like in the case of $\mathrm{LiF}$ for $\theta_{i}=0.7^{\circ}$.

In order to analyze the origin of channeling effects, we made a statistical study of the trajectories. Figure 4(a) shows a random set of reflected trajectories for protons impinging on $\mathrm{LiF}$ with $\theta_{i}=0.3^{\circ}$. We found that differences between channeling and random directions for very glancing angles,

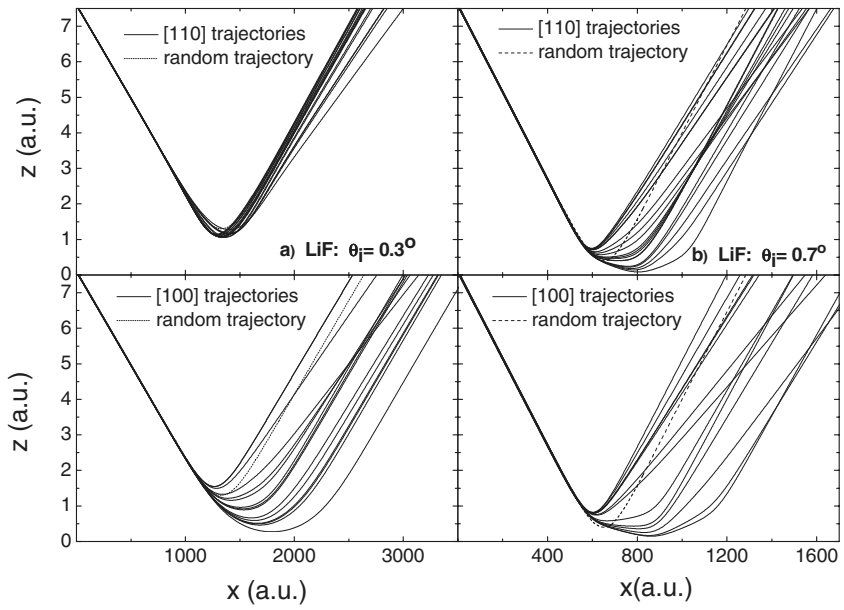

FIG. 4. Random set of trajectories corresponding to the results of (a) Fig. 1 (left two graphs) and (b) Fig. 2 (right two graphs). 
for which the penetration is negligible, are mainly due to the major approximation of the whole trajectory to the insulator surface in the case of channeling along the [100] direction. This is in accord with the explanation given in the experimental work of Andou et al. [6]. Note that for the more open [100] channel the spread of the trajectories is larger than for the [110] channel, giving rise to a considerable variation in the closest distance to the surface. For the [100] channel most of the trajectories display distances of maximum approach to the surface significantly smaller than the corresponding to random incidence, which produces a noticeable increment of the electron emission.

However, when the impact angle becomes higher, the spreads of trajectories and minimum distances to the surface are very similar for both channels, as can be appreciated in Fig. 4(b). For the incidence angle $\theta_{i}=0.7^{\circ}$, for which the penetration rate is high, although the spreads of channeled trajectories are large, deviations of closest distances to the surface with respect to the random values are smaller than 0.5 a.u., which reduces strongly the enhancement of the channeled electron emission probability for nonpenetrating projectiles, as experimentally observed by Winter et al. [5].

Something similar happens for $\mathrm{KCl}$ surfaces. As mentioned, the incidence angle corresponding to the experiment of Andou et al. $\theta_{i}=0.28^{\circ}$ presents a significant penetration rate within our punctual model. However, a more precise description of the surface-projectile interaction, taking into account surface effects, might modify the proportion of projectiles penetrating into the solid, affecting present results.

\section{CONCLUSION}

The penetration of projectiles into the solid surface must be considered as another important ingredient in the analysis of channeling effects on the electron emission probability. For very grazing projectiles, where the penetration rate is negligible, we found an enhancement of the electron emission yield for incidence along the most opened [100] channel. Differences between channeling and random directions are mainly due to the major approximation of the whole trajectory to the insulator surface in the case of channeling. But when the incidence angle increases and the penetration becomes important, channeling effects corresponding to penetrating projectiles steeply diminish. For the [100] channel the electron emission yield approaches the random value, being close to the emission rate for the [110] channel. This is because the spreads of trajectories and minimum distances to the surface become similar for both channeling directions, reducing the differences between the corresponding emission probabilities. Therefore, we conclude that the phenomena of penetration affects electron emission even for those trajectories recorded in coincidence with specularly reflected projectiles, diminishing the differences between electron emission probabilities for [100] and random incidences as the penetration rate increases.
[1] H. Winter, Phys. Rep. 367, 387 (2002).

[2] R. Sizmann and C. Varelas, Nucl. Instrum. Methods 132, 633 (1976).

[3] H. J. Andrä, H. Winter, R. Fröhling, N. Kirchner, H. J. Plön, W. Wittmann, W. Graser, and C. Varelas, Nucl. Instrum. Methods 170, 527 (1980).

[4] M. Hasegawa, K. Kimura, Y. Fujii, M. Suzuki, Y. Susuki, and M. Mannami, Nucl. Instrum. Methods Phys. Res. B 33, 334 (1988).

[5] H. Winter, K. Maass, S. Lederer, H. P. Winter, and F. Aumayr, Phys. Rev. B 69, 054110 (2004).

[6] G. Andou, K. Nakajima, and K. Kimura, Nucl. Instrum. Methods Phys. Res. B 160, 16 (2000).

[7] K. Kimura, S. Ooki, G. Andou, K. Nakajima, and M. H. Man- nami, Phys. Rev. A 58, 1282 (1998).

[8] K. Kimura, G. Andou, and K. Nakajima, Phys. Rev. Lett. 81, 5438 (1998).

[9] M. S. Gravielle, I. Aldazabal, A. Arnau, V. H. Ponce, J. E. Miraglia, F. Aumayr, S. Lederer, and H. Winter, Phys. Rev. A 76, 012904 (2007).

[10] A. J. Garcia and J. E. Miraglia, Phys. Rev. A 74, 012902 (2006).

[11] P. D. Fainstein, V. H. Ponce, and R. D. Rivarola, J. Phys. B 24, 3091 (1991).

[12] M. S. Gravielle, Phys. Rev. A 62, 062903 (2000).

[13] A. Arnau, M. S. Gravielle, J. E. Miraglia, and V. H. Ponce, Phys. Rev. A 67, 062902 (2003). 